\title{
Update on the management of inflammatory bowel disease: specific role of adalimumab
}

This article was published in the following Dove Press journal:

Clinical and Experimental Gastroenterology

14 July 201 I

Number of times this article has been viewed

\section{Luisa Guidi \\ Daniela Pugliese \\ Alessandro Armuzzi \\ Internal Medicine and Gastroenterology, Complesso Integrato Columbus, Università Cattolica del Sacro Cuore, Rome, Italy}

Correspondence: Luisa Guidi UO Medicina interna e Gastroenterologia, Complesso integrato Columbus,

Via Moscati 3I, 00I68 Roma, Italy

Tel +3963503239

Fax +39 6305464l

Email lguidi@rm.unicatt.it
Abstract: Anti-tumor necrosis factor alpha (TNF- $\alpha$ ) medications are a class of biologics employed in the treatment of patients with inflammatory bowel disease (IBD). Adalimumab is the first fully human monoclonal immunoglobulin directed against TNF- $\alpha$, which binds with high affinity and specificity to membrane and soluble TNF. Adalimumab administered subcutaneously has demonstrated efficacy in the treatment of rheumatoid arthritis, ankylosing spondylitis, psoriatic arthritis, and severe chronic psoriasis. Studies have shown that adalimumab is effective for inducing and maintaining remission of moderate-to-severe active Crohn's disease (CD) patients at an induction dose of 160/80 $\mathrm{mg}$ (week 0 and 2) and at a maintenance dose of $40 \mathrm{mg}$ every other week. The efficacy of adalimumab as a second-line therapy has also been documented for patients with loss of response or intolerance to infliximab. Adalimumab is also superior to placebo for inducing and maintaining complete perianal fistula closure. It also seems effective for reducing extraintestinal manifestations. The safety profile is similar to that of other anti-TNF therapy in CD patients, with lower immunogenicity and rate of adverse injection reactions than infliximab. Adalimumab is not approved for the treatment of ulcerative colitis (UC). Recently, however, the results of the first randomized, controlled trial on adalimumab for UC showed that adalimumab at 160/80 mg induction dose was safe and effective for inducing remission and clinical response after 8 weeks in patients with moderately-to-severely active UC failing treatment with corticosteroids and/or immunosuppressants. More data are necessary to clarify the therapeutic role of adalimumab in UC. This review of the literature summarizes available data on the efficacy and safety profile adalimumab in patients with IBD.

Keywords: anti-TNF- $\alpha$, adalimumab, Crohn's disease, ulcerative colitis

\section{Introduction}

Inflammatory bowel diseases (IBD) are lifelong intestinal inflammatory conditions of unknown etiology, characterized by remissions and exacerbations. The main phenotypes of IBD are Crohn's disease (CD) and ulcerative colitis (UC). Their pathogenesis is not totally understood, but in both diseases, bowel damage is induced by an uncontrolled activation of both innate and adaptive immunity due to an inappropriate immune response to luminal antigens. This mechanism results in an imbalance between pro-inflammatory cytokines - interferon gamma (IFN- $\gamma$ ), tumor necrosis factor alpha (TNF- $\alpha$ ), interleukin-1 (IL-1), IL-12 - and anti-inflammatory mediators, which maintains chronic tissue damage. TNF- $\alpha$, produced by macrophages and activated $\mathrm{T}$ cells, plays a key role inducing further stimulation and recruitment of other inflammatory cells. ${ }^{1}$ 
Treatment decisions for IBD are typically based on disease severity (mild, moderate, and severe) and extension. Over recent years, the management of IBD has dramatically changed. In particular, advances in understanding the pathogenesis and the natural course of the disease have substantially changed the therapeutic algorithms with the introduction of new biological drugs. Among these the anti-TNF- $\alpha$ monoclonal antibodies infliximab and adalimumab are currently approved for the management of $\mathrm{CD}$, in particular for patients with moderately and severely active luminal disease who are nonresponders to conventional therapy. A third anti-TNF- $\alpha$ agent, certolizumab pegol, is approved for CD only in the US, Switzerland, and Russia. Infliximab and adalimumab are also indicated in the setting of surgical procedures as the first-choice medical therapy for complex perianal $\mathrm{CD}^{2,3}$ At present only infliximab is approved for UC and is recommended for patients with moderate and severe UC refractory to corticosteroids and/ or immunomodulators. ${ }^{4,5}$ Adalimumab is a fully human anti-TNF- $\alpha$ monoclonal antibody and consists of humanderived heavy- and light-chain variable regions and human IgG1 constant region. Adalimumab binds specifically to TNF- $\alpha$ and blocks its interaction with the $\mathrm{p} 55$ and $\mathrm{p} 75$ cell surface TNF receptors. It also lyses surface TNF-expressing cells in vitro in the presence of complement, but does not bind or inactivate lymphotoxin (TNF- $\beta$ ). It is administered subcutaneously (allowing home-based care) with induction dosing of $160 \mathrm{mg}$ followed by $80 \mathrm{mg} 2$ weeks later (mean serum adalimumab trough concentrations of approximately $12 \mu \mathrm{g} / \mathrm{mL}$ at week 2 and week 4). Subsequent maintenance dosing is of $40 \mathrm{mg}$ every other week (eow). The pharmacokinetics of adalimumab were linear over the dose range of 0.5 to $10.0 \mathrm{mg} / \mathrm{kg}$ following a single intravenous dose. ${ }^{6}$

\section{Adalimumab in Crohn's disease Luminal CD}

In the CLASSIC 1 trial (a 4-week multicenter, randomized, placebo-controlled study), 299 patients with moderate-to-severe active luminal CD, naïve to anti-TNF- $\alpha$, received induction therapy with adalimumab $40 \mathrm{mg} / 20 \mathrm{mg}$, $80 \mathrm{mg} / 40 \mathrm{mg}, 160 \mathrm{mg} / 80 \mathrm{mg}$, or placebo, at weeks 0 and 2 . The primary endpoint was remission at week 4 defined by a $\mathrm{CD}$ activity index $(\mathrm{CDAI})<150$. At week 4 , the remission rate was significantly higher in the $160 / 80 \mathrm{mg}$ adalimumab group compared with placebo ( $36 \%$ vs $12 \%, P<0.001$ ). Clinical response (defined as a reduction of 70 points in the CDAI) was demonstrated in both higher dose adalimumab arms (80/40 mg: 59\%, $P<0.05 ; 160 / 80 \mathrm{mg} 59 \%, P<0.01$ vs placebo 37\%). ${ }^{7}$ At week 4, from the same cohort, 276 patients in clinical remission (CDAI $<150$ points) were enrolled in the CLASSIC 2 trial. ${ }^{8}$ After 4 open-label weeks of administration of adalimumab eow, 55 patients in clinical remission were re-randomized to receive blinded adalimumab maintenance treatment with $40 \mathrm{mg}$ eow or $40 \mathrm{mg}$ weekly or placebo for 56 weeks. Patients who were not in clinical remission continued open-label adalimumab eow, with the option of increasing their dosage in case of flare or nonresponse. In the randomized arm, patients who experienced flares or nonresponse could switch to the open-label adalimumab eow arm. Remission rates were significantly higher for both the active arms than for placebo (40 mg eow: 79\% 15/19 patients, 40 mg weekly: $83 \% 15 / 18$ vs placebo: $44 \% 8 / 18, P<0.05$ ). Patients who received either dosage of adalimumab also had a numerically greater rate of clinical response (reduction of 100 and 70 points in the CDAI score) compared with patients who received placebo. In the open-label arm, 46\% patients achieved remission at 56 weeks. The rates of serious adverse events were low in patients treated with adalimumab and were similar to placebo. Data from the CHARM trial, ${ }^{9}$ conducted in $\mathrm{CD}$ patients with the same clinical characteristics, support in a larger cohort (854 patients) the efficacy of adalimumab over placebo for maintaining clinical remission at 26 and 56 weeks. In this trial, patients, after an open-label induction regimen of adalimumab $80 / 40 \mathrm{mg}$, were randomized at week 4 to receive adalimumab $40 \mathrm{mg}$ eow, $40 \mathrm{mg}$ weekly, or placebo. Patients who experienced a disease flare (increase in CDAI of $\geq 70$ points vs week 4 and an absolute CDAI score $>220$ ) or sustained nonresponse (those who did not attain a decrease in CDAI of $\geq 70$ points vs baseline) at week 12 were subsequently permitted to switch to open-label adalimumab $40 \mathrm{mg}$ eow therapy. Open-label adalimumab patients with continued nonresponse or flares with $40 \mathrm{mg}$ eow could switch to $40 \mathrm{mg}$ weekly. Remission rates were significantly higher for both the active arms than for placebo at week $26(40 \mathrm{mg}$ eow: $40 \%, 40$ mg weekly: $47 \%$ vs placebo: $17 \%, P<0.001)$ and week 56 (36\% and $41 \%$, vs $12 \%$ placebo, $P<0.001)$. The proportion of patients in remission was significantly different between the active arms and placebo since week 6 and through week 56. No significant differences were found between the dosage of $40 \mathrm{mg}$ eow and $40 \mathrm{mg}$ weekly. Concomitant immunomodulators or previous anti-TNF therapy did not affect response, remission, or steroid withdrawal. However, among patients without concomitant immunosuppressants, the group treated with weekly adalimumab injections had 50\% remission rate vs 33\% eow, but this difference was not significant. Among all randomized patients 35\% 
withdrew before the end of treatment and 50\% moved to unblinded adalimumab eow. The subgroup analysis showed that adalimumab was superior to placebo for maintaining a steroid-free clinical remission at 26 (adalimumab $40 \mathrm{mg}$ eow $35 \%, 40 \mathrm{mg}$ weekly $30 \%$ vs placebo $6 \%, P<0.001$ for each adalimumab group) and 56 weeks (adalimumab $40 \mathrm{mg}$ eow $29 \%, 40 \mathrm{mg}$ weekly $23 \%$ vs placebo $3 \%, P<0.001$ only for adalimumab eow). Hospitalization risks for all and CD-related causes were also higher for patients who received placebo compared with adalimumab. Fewer CD-related surgeries occurred in the adalimumab groups (eow, weekly, and combined groups) compared with placebo $(0.4,0.8$, and 0.6 vs 3.8 per 100 patients; all $P<0.05) .{ }^{10}$ Post hoc intention-totreat analysis, including all patients randomized regardless of whether they switched to the open-label arm, revealed also that continuous therapy with adalimumab was superior for inducing clinical remission compared with induction only or restart in case of flare (51\% eow and $49 \%$ weekly, vs 38\%, $P<0.05)$. The combined continuous adalimumab treatment group had also a $52 \%$ reduction in the number of surgeries $(P<0.01)$, fewer flares, and less risk of hospitalizations than the induction/re-initiation group $(P<0.05) .{ }^{11}$ Of the 260 patients initially randomized to adalimumab eow, $46 \%$ switched to open-label treatment because they experienced flares or lack of response. Among these, 19\% moved to openlabel eow therapy and $27 \%$ moved to open-label weekly. From a regression model evaluating baseline characteristics, the CDAI score was the only statistically significant predictor of dose escalation, with a $72 \%$ greater risk for weekly dosing when baseline CDAI increases by 100 points. ${ }^{12}$ At 56 weeks, 467 patients who completed the CHARM study entered the extension open-label study (ADHERE). All blinded patients received adalimumab eow, while patients who had already moved to open-label adalimumab eow or weekly continued their regimens upon entry in ADHERE. After up to 2 years of therapy, $37.6 \%, 41.9 \%$, and $49.8 \%$ of patients originally randomized to placebo, adalimumab eow, and adalimumab weekly, respectively, were in clinical remission. All groups experienced sustained improvements in health-related quality life (measured with Inflammatory Bowel Disease Questionnaire [IBDQ]). ${ }^{13}$

In the large cohort of the open-label CARE study, 945 patients received 160/80 mg adalimumab at week 0-2 and then $40 \mathrm{mg}$ eow. The percentage of patients in remission in the adalimumab group at week 4 was $43 \%$ and increased to $52 \%$ at week 20 . From the same cohort, among the 497 patients with baseline extraintestinal manifestation, $51 \%$ of patients were free of symptoms and signs at week $20 .{ }^{14}$
Recently, data from a systematic review on the loss of response and need for adalimumab dose intensification in adult and pediatric CD were published. Thirty-nine studies were included. The mean percentage of patients who lost response to adalimumab was $18.2 \%$ among a total of 955 primary responders, with a calculated annual risk of $20.3 \%$ per patient-year. The mean percentage of patients who needed an adalimumab dose escalation was $21.4 \%$ and the annual risk was $24.4 \%$ per patient-year. Adalimumab dose escalation permitted response to be regained in $71.4 \%$ and remission in $39.9 \%$ of patients. Several predictors for loss of response were identified, but only previous treatment with anti-TNF therapy was confirmed in multiple studies. ${ }^{15}$

The results from the described randomized, controlled trials (RCT) are summarized in Table 1.

\section{Perianal fistulizing CD}

A post hoc analysis of the CHARM trial, on the efficacy in healing of draining perianal fistulas, has shown that adalimumab induced a reduction in the mean number of draining fistulas per day compared with placebo $(0.88 \mathrm{vs}$ 1.34, $P<0.002$ ) during the 1 -year double-blind treatment period. About $90 \%$ of patients who healed fistulas at the end of the CHARM trial maintained long-term fistula healing on 1 year of open-label therapy. ${ }^{16}$ In the open-label single arm CHOICE trial, $673 \mathrm{CD}$ patients, who were infliximab primary nonresponders $(17 \%)$ or initial responders $(83 \%)$, were enrolled and treated with adalimumab (induction dose of 160/80 mg respectively at week 0 and 2, maintenance dose $40 \mathrm{mg}$ eow) after a minimum of 8 weeks of infliximab washout period. At baseline, 88 patients $(13 \%)$ had at least one draining cutaneous fistula. Complete fistula healing was achieved by 34 patients (39\%) at the last visit (dates ranged from week 4 to week 36). In particular, 29.4\% (10 of 34) completed week 12 and 64.7\% (22 of 34) completed week 24 or week 36. Improvements in quality of life and work productivity were sustained from week 4 to week 24 for all patients, as well as the subgroup of primary nonresponders. ${ }^{17}$

\section{CD patients with primary or secondary nonresponse to infliximab}

Sandborn et al, in the GAIN study, reported the first data on adalimumab use in infliximab intolerant or secondary nonresponder patients. ${ }^{18}$ In this 4 -week trial, 325 patients with moderate-to-severe active CD (CDAI 220 to 450 points) were randomized to receive blinded 160/80 mg adalimumab at week 0 and 2 or placebo. Patients were classified as: "having loss of response", if they had received 
Table I Randomized controlled trials on adalimumab in Crohn's disease

\begin{tabular}{|c|c|c|c|c|}
\hline & No. of patients & Treatment & \multicolumn{2}{|l|}{ Primary outcomes } \\
\hline \multirow[t]{6}{*}{ Hanauer et $\mathrm{al}^{7}$} & 299 & & \multicolumn{2}{|l|}{ Week $4 \%$ ( $P$ vs placebo) } \\
\hline & & Induction: & \multicolumn{2}{|l|}{ Remission rate } \\
\hline & & $40 \mathrm{mg} / 20 \mathrm{mg}$ week $0-2$ & \multicolumn{2}{|l|}{$18(0.36)$} \\
\hline & & $80 \mathrm{mg} / 40 \mathrm{mg}$ week $0-2$ & \multicolumn{2}{|l|}{$24(0.06)$} \\
\hline & & 160 mg/80 mg week 0-2 & \multicolumn{2}{|l|}{$36(0.001)$} \\
\hline & & Placebo week 0-2 & \multicolumn{2}{|l|}{12} \\
\hline \multirow[t]{5}{*}{ Sandborn et $\mathrm{al}^{8}$} & 276 & & \multicolumn{2}{|l|}{ Week $56 \%$ ( $P$ vs placebo) } \\
\hline & & $55^{\mathrm{a}}$ maintenance: & \multicolumn{2}{|l|}{ Remission rate } \\
\hline & & $40 \mathrm{mg}$ eow for 56 weeks & \multicolumn{2}{|l|}{$79(0.05)$} \\
\hline & & $40 \mathrm{mg}$ weekly for 56 weeks & \multicolumn{2}{|l|}{$83(0.05)$} \\
\hline & & Placebo & \multicolumn{2}{|l|}{44} \\
\hline \multirow[t]{4}{*}{ Colombel et $\mathrm{al}^{9}$} & 854 & $778^{\mathrm{b}}$ maintenance: & \multicolumn{2}{|l|}{ Week $26 \%$ ( $P$ vs placebo) } \\
\hline & & $40 \mathrm{mg}$ eow for 56 weeks & $40(0.001)$ & $36(0.00 I)$ \\
\hline & & $40 \mathrm{mg}$ weekly for 56 weeks & $47(0.001)$ & $41(0.00 I)$ \\
\hline & & Placebo & 17 & 12 \\
\hline \multirow[t]{5}{*}{ Colombel et al ${ }^{16}$} & 117 & II $7^{c}$ maintenance: & \multicolumn{2}{|c|}{ Week 56 mean number of draining fistula } \\
\hline & & $40 \mathrm{mg}$ eow for 56 weeks & \multicolumn{2}{|l|}{0.85} \\
\hline & & $40 \mathrm{mg}$ weekly for 56 weeks & \multicolumn{2}{|l|}{0.91} \\
\hline & & Both adalimumab group for 56 weeks & \multicolumn{2}{|l|}{$0.88(P<0.002$ vs placebo $)$} \\
\hline & & Placebo & \multicolumn{2}{|l|}{1.34} \\
\hline \multirow[t]{4}{*}{ Sandborn et al ${ }^{18}$} & 325 & & \multicolumn{2}{|l|}{ Week $4 \%$ ( $P$ vs placebo) } \\
\hline & & Induction: & \multicolumn{2}{|l|}{ Remission rate } \\
\hline & & $160 \mathrm{mg} / 80 \mathrm{mg}$ week $0-2$ & \multicolumn{2}{|l|}{$21(P<0.001)$} \\
\hline & & Placebo & \multicolumn{2}{|l|}{7} \\
\hline \multirow[t]{4}{*}{ Colombel et al ${ }^{21}$} & 135 & & \multicolumn{2}{|r|}{ Week 52} \\
\hline & & $129^{\mathrm{d}}$ maintenance: & Remission rate \% & ( $P$ vs placebo) \\
\hline & & $40 \mathrm{mg}$ eow & $16(0.34)$ & 19 \\
\hline & & Placebo & 10 & 0 \\
\hline Hyams et $\mathrm{a}^{25}$ & 192 & I88e maintenance for 48 weeks: & Clinical remission week 26 & \\
\hline & & High dose $(40$ mg eow BW > 40 kg, & High dose $39 \%$ vs low dos & $\%(P=0.075)$ \\
\hline & & $20 \mathrm{mg}$ eow $\mathrm{BW}<40 \mathrm{~kg}$ ) & & \\
\hline & & Low dose $(20 \mathrm{mg}$ eow BW $>40 \mathrm{~kg}$, & & \\
\hline & & $10 \mathrm{mg}$ eow $\mathrm{BW}<40 \mathrm{~kg}$ ) & & \\
\hline
\end{tabular}

Notes: aln remission at week 0 and 2 after 2 open-label $40 \mathrm{mg}$ adalimumab doses; bafter an open-label induction $80 / 40 \mathrm{mg}$ at week 0 and 2 ; ${ }^{\mathrm{c}}$ patients with draining fistula at baseline in the cohort of the CHARM trial; after an open-label induction 160/80 mg at week 0-2; eafter an open-label adalimumab induction per body-weight ( $160 \mathrm{mg} / 80 \mathrm{mg}$ week $0-2$ if body weight $>40 \mathrm{~kg}, 80 \mathrm{mg} / 40 \mathrm{mg}$ week $0-2$ if body weight $<40 \mathrm{~kg}$ ).

Abbreviation: eow, every other week.

at least 2 doses of infliximab and after an initial response, they lacked improvement, or had clinical worsening at least 2 weeks after the last dose of infliximab; "intolerant", if they had an acute adverse reaction or delayed infusion reaction. At 4 weeks, adalimumab induced higher rates of remission compared with placebo $(21 \%$ vs $7 \%, P<0.001)$. The improvements in CDAI by $70-100$ points were also higher in the adalimumab arm. Patients in the adalimumab arm had statistically significantly lower mean CDAI total scores at week 1, 2, and 4 than did patients in the placebo group. At the end, patients who completed this trial were able to continue in the open-label extension and receive adalimumab $40 \mathrm{mg}$ eow. Remission rate was $39 \%$ at 6 months and $29 \%$ at 1 year. ${ }^{19}$ Data on the efficacy of adalimumab in primary nonresponders to infliximab are limited. Among the 945 patients of the open-label cohort (OLC) of the CARE study, about one-quarter of patients were primary nonresponders to infliximab. Remission rate was significantly different between infliximab-naive and infliximab-exposed patients (62\% vs $42 \%, P<0.001)$. No significant differences in remission rates were found between primary nonresponders and intolerant patients or patients having loss of response. ${ }^{14}$ A systematic review including all the OLC and RCT was performed by Ma et al, ${ }^{20}$ evaluating the efficacy of adalimumab in infliximab primary or secondary nonresponders. From the 15 studies (2 RCT and 13 OLC) included, $1810 \mathrm{CD}$ patients with previous infliximab were identified. Short-term clinical response at 4 weeks ranged from $41 \%$ to $83 \%$. Long-term clinical remission at 12 months ranged from $19 \%$ to $68 \%$. The authors concluded that the observed variability was due to differences in the study design and baseline characteristics of patients included in the studies. 


\section{Mucosal healing}

Colombel et al recently presented data from the EXTEND study (the available data are limited, because only the abstract is available). ${ }^{21}$ In this randomized placebo-controlled study, 135 patients with moderate-to-severe ileocolonic $\mathrm{CD}$ (CDAI, 220-450) and baseline mucosal ulceration (score of 2 or 3 for $\geq 1$ colon segment in the ulcerated surface subscore of the Simple Endoscopic Score for Crohn's disease) were enrolled. After an open-label adalimumab induction therapy with $160 \mathrm{mg}$ at week 0 and $80 \mathrm{mg}$ at week 2, 129 patients were randomized to maintenance therapy with adalimumab $40 \mathrm{mg}$ eow or placebo. Patients who had flares or did not respond could receive open-label adalimumab from week 8 . The primary endpoint was deep remission, defined as clinical remission with CDAI of $<150$, plus mucosal healing, as evidenced by the absence of mucosal ulceration at week 12 and 52. At week 12, more patients in the adalimumab group (16\%) than in the induction-only placebo group (10\%) had achieved both clinical remission and mucosal healing, although the difference was not statistically significant $(P=0.34)$. At week 52 , however, there was a highly significant difference between the two groups (19\% vs $0 \%$ for adalimumab and placebo, respectively; $P<0.001)$. Post hoc analysis revealed that early deep remission predicts better long-term outcomes. ${ }^{22}$ Patients with early deep remission were about five times more likely to achieve both IBDQ remission and a normal SF-36 physical (PCS) score (both $P<0.05$ ) at week 52 .

\section{Pediatric CD}

The RESEAT study is a large multicenter experience using adalimumab for pediatric $\mathrm{CD} .{ }^{23}$ In this retrospective study, data on 115 children, treated with adalimumab are reported. Overall, $65 \%$ of all subjects had moderate-to-severe disease activity at baseline by physician global assessment (PGA), whereas $21 \%$ had mild disease and $15 \%$ were inactive. Mean age at the time of diagnosis of $\mathrm{CD}$ was $11.1 \pm 3.1$ years and the first dose of adalimumab was administered at a mean age of $15.8 \pm 3.0$ years, which was equivalent to a mean of $4.7 \pm 2.8$ years (range $0.3-14.7$ years) after diagnosis. Only $5 \%$ (6) of patients were naïve to infliximab. Reasons for the discontinuation of infliximab therapy included secondary loss of response to the agent in 51 patients (47\%), infusion reaction or delayed hypersensitivity in $49(45 \%)$, and preference for an injectable agent in ten patients $(9 \%)$. The clinical response rates at 3, 6, and 12 months after the start of adalimumab therapy were $65 \%, 71 \%$, and $70 \%$, measured with PGA (clinical response was defined as a decrease in the PGA from either moderate or severe to mild/inactive or from mild to inactive).
Clinical remission (improvement in PGA to inactive) was $32 \%, 43 \%$, and $49 \%$, at 3,6 , and 12 months, respectively. There were no malignancies, serious infections, or deaths in the study subjects. Recently, Russell et al reported data of a multicenter English cohort of $70 \mathrm{CD}$ children with a median age of 14.8 years and a median duration of disease of 4.3 years at start of adalimumab treatment. Among these, $91 \%$ had prior thiopurines and $94 \%$ had prior infliximab usage. Remission rates were $24 \%, 58 \%$, and $41 \%$ at 1,6 , and 12 months, respectively, with higher rate of remission in the subgroup of patients on concomitant immunosoppression with thiopurine or methotrexate (34/46 [74\%] vs $9 / 24$ [37\%], $P=0.003)$. In this cohort, 15 adverse events were recorded and four of these were serious (6\%), including one severe case of Clostridium difficile infection, one case of stomal abscess with subsequent fistulization, and two deaths due to sepsis ( $3 \%$ mortality rate). Both the patients who died were receiving adalimumab plus concomitant immunosuppressive drugs and parenteral nutrition. ${ }^{24}$ Recently, data on the first randomized, double-blind trial on the use of adalimumab in children were reported. ${ }^{25}$ In this study, 192 children, after an open-label induction per body weight $(160 \mathrm{mg} / 80 \mathrm{mg}$ week $0-2$ if body weight $>40 \mathrm{~kg}$, $80 \mathrm{mg} / 40 \mathrm{mg}$ week $0-2$ if body weight $<40 \mathrm{~kg}$ ), stratified for previous infliximab exposure and for week 4 response, were randomized at week 4 to receive adalimumab maintenance therapy at high dose ( $40 \mathrm{mg}$ eow if body weight $>40 \mathrm{~kg}$ or $20 \mathrm{mg}$ eow if body weight $<40 \mathrm{~kg}$ ) or at low dose $(20 \mathrm{mg}$ eow if body weight $>40 \mathrm{~kg}$ or $10 \mathrm{mg}$ eow if body weight $<40 \mathrm{~kg}$ ) for 48 weeks. At week 26, a higher proportion in the high-dose group achieved clinical remission than in the low-dose group (39\% vs $28 \%, P=0.075$ ), with a significant difference between Infliximab naïve patients $(P=0.026)$ and those with clinical response at week $4(P=0.016)$.

\section{Adalimumab in ulcerative colitis}

Adalimumab is not approved to treat UC patients. The first evidence for the efficacy of adalimumab in UC, from openlabel trials ${ }^{26-30}$ and retrospective studies, ${ }^{31,32}$ revealed that adalimumab could be an effective therapeutic option for inducing and maintaining remission in patients with active UC intolerant or refractory to standard therapy.

However, the results of a double-blind RCT were recently published. It was an 8-week multicenter study, conducted in North America and Europe and included patients with moderately-to-severely active UC (full Mayo score of 6-12, with endoscopic subscore of 2-3). ${ }^{33}$ All patients were naïve to anti-TNF and failed or did not tolerate standard treatments with corticosteroids or immunomodulators. Initially 
186 patients were enrolled and randomized to subcutaneous treatment with an induction dose of 160/80 $\mathrm{mg}$ or placebo. Subsequently, after an amendment (number 3 ) to the protocol, a third arm receiving adalimumab at induction dose of 80/40 mg was included. Primary endpoint was clinical remission at week 8 (full Mayo Score $\leq 2$ ). Secondary endpoints were clinical response, mucosal healing, and improvement in rectal bleeding, PGA, and decrease in stool frequency at week 8. At week 8 , the proportion of patients in clinical remission in the adalimumab $160 / 80 \mathrm{mg}$ group was $18.5 \%$ compared with $9.2 \%$ of the placebo group $(P=0.031)$ with a therapeutic gain of adalimumab over placebo of $9.3 \%$. No difference was found between the adalimumab $80 / 40 \mathrm{mg}$ group (10\% remission rate) and placebo group $(P=0.833)$. There was a higher proportion of patients with subscores of mild disease in the adalimumab 160/80 mg group compared with placebo, with significant differences for the rectal bleeding subscore $(P=0.038)$ and PGA $(P=0.035)$. No significant differences were found in terms of clinical response and mucosal healing between the three arms. In particular the clinical response was obtained in $44.6 \%$ of patients in the placebo arm, in $51.5 \%$ of patients in the adalimumab $80 / 40 \mathrm{arm}$, and in $54.6 \%$ of patients in the adalimumab 160/80 arm. The mucosal healing was scored in $41.5 \%$ of patients in the placebo arm, in $37.7 \%$ of patients in the adalimumab $80 / 40$ arm, and in $54.6 \%$ of patients in the adalimumab 160/80 arm. The additional post hoc analyses showed that extensive disease, more active disease, and high levels of C-reactive protein $(\mathrm{CRP}=10 \mathrm{mg} / \mathrm{L})$ were associated with low rates of clinical remission, which possibly reflects a greater efficacy of adalimumab in less severe disease. In the subgroup of patients who weighed $>82 \mathrm{~kg}$, remission rate was lower than in patients who weighed $<70 \mathrm{~kg}$, especially in the adalimumab $160 / 80 \mathrm{mg}$ group (9.6\% vs $24.4 \%$, respectively). After 8 weeks, 390 patients (originally randomized to all 3 arms) entered an open-label extension to receive adalimumab
$40 \mathrm{mg}$ eow as maintenance therapy through 52 weeks. In case of flare or nonresponse, the dose could be increased to weekly. At 52 weeks, $25.6 \%$ of patients were in clinical remission with maintenance therapy of $40 \mathrm{mg}$ eow (dose escalation considered as failure). From a post hoc analysis, not taking into account patients who dose escalated as failures, remission rate at 52 weeks was $29.5 \%{ }^{34}$ The quality of life through 52 weeks, measured by IBDQ, has been improved by adalimumab maintenance therapy in UC. ${ }^{35}$

Recently, the results of an induction and maintenance study were presented. ${ }^{36}$ In this study 494 UC patients (Mayo score 6-12, endoscopic subscore 2-3 points) were randomized to placebo or adalimumab $160 \mathrm{mg}$ at week $0,80 \mathrm{mg}$ at week 2 , and then $40 \mathrm{mg}$ eow starting on week 4. Co-primary endpoints were clinical remission at week 8 and clinical remission at week 52 . Significantly more adalimumab-treated patients achieved clinical remission at week $8(16.55 \%$, $P=0.02)$ and at week $52(17.3 \%, P<0.01)$ compared with placebo (9.3\% at week 8 and $8.5 \%$ at week 52$)$. Adalimumab was efficacious in inducing and maintaining clinical remission in patients with moderate-to-severe UC who did not respond adequately to conventional therapy with oral corticosteroids/immunosuppressants.

The results from the described RCTs are summarized in Table 2.

\section{Safety}

Because adalimumab is administered subcutaneously, the injection site reactions are generally mild and do not necessitate drug discontinuation. ${ }^{6}$ This is a major advance compared with infliximab, which is administered through an infusion, which could be complicated by acute adverse reactions, including mild to severe clinical manifestations and delayed hypersensitivity-like reactions. The safety profile of adalimumab in global clinical trials in CD patients

Table 2 Randomized controlled trials on adalimumab in ulcerative colitis

\begin{tabular}{|c|c|c|c|}
\hline & No. of patients & Treatment & Outcomes \\
\hline \multirow[t]{4}{*}{ Reinisch et $\mathrm{al}^{33}$} & 576 & Induction: & Clinical remission \\
\hline & & 160 mg/80 mg week 0-2 & $18.5(0.03 \mid)$ \\
\hline & & Placebo week 0-2 & $9.2(0.833)$ \\
\hline & & $80 \mathrm{mg} / 40 \mathrm{mg}^{\mathrm{a}}$ week $0-2$ & 10 \\
\hline \multirow[t]{7}{*}{ Sandborn et $\mathrm{al}^{36}$} & 494 & Induction: & Clinical remission \\
\hline & & 160 mg/80 mg week 0-2 & $\%$ ( $P$ vs placebo) week 8 \\
\hline & & Placebo week 0-2 & $16.5(0.02)$ \\
\hline & & & 9.3 \\
\hline & & Maintenance: & $\%$ (P vs placebo) week 52 \\
\hline & & $40 \mathrm{mg}$ eow starting at week 4 & $17.3(<0.01)$ \\
\hline & & Placebo week 0-2 & 8.5 \\
\hline
\end{tabular}

Note: ${ }^{a}$ This arm was included after amendment 3 , adding a third arm of adalimumab at induction, with a dose of $80 / 40 \mathrm{mg}$. Abbreviation: eow, every other week. 
was similar to that of other TNF antagonists in CD and the rates of adverse events were comparable to those of the other approved indications for adalimumab. Analyzing the largest trial of adalimumab in $\mathrm{CD}$, the types and frequency of adverse events, serious adverse events, and adverse events of interest were similar to placebo during both induction and maintenance periods and did not differ by dosing regimen used during induction or maintenance. ${ }^{37}$ No difference was found for serious infections between placebo and the 2 adalimumab groups (40 mg eow and $40 \mathrm{mg}$ weekly). ${ }^{7,8}$ Of 172 patients in the CLASSIC 2 cohort with measurement at baseline and at 56 weeks and no antinuclear antibodies at baseline, $33(19 \%)$ tested positive at their final visits and anti-ds-DNA antibodies were observed in all 33 patients at this time point. None of these patients developed novel clinical symptoms suggestive of autoimmunity. ${ }^{8}$ In the allexposed patients from the main trials, 56 patients $(1.8 \%)$ had opportunistic infections. Oral candidiasis/thrush (37 patients, $1.2 \%$ ) was the most commonly reported opportunistic infection). The most frequently reported serious adverse event was serious infection (5.8\%), most commonly abscess (intra-abdominal and gastrointestinal-related), followed by gastrointestinal infections (excluding abscess), and pulmonary infections. Low incidences of malignancies were reported in the $\mathrm{CD}$ trials, in particular one lymphoma (0.07/100 patients year). Lupus-related disorders and demyelinating disorders, although uncommon, occurred in adalimumab-treated patients with CD. Four deaths were recorded; in particular only one of these was related to adalimumab therapy (one case of acute myeloid leukemia). ${ }^{37}$ As with all other anti-TNF- $\alpha$ drugs having an impact on cell-mediated immunity, there is a warning on the emerging infections sustained by intracellular pathogens (such as tuberculosis, listeriosis, and histoplasmosis). Therefore, screening for latent tuberculosis should be performed before starting adalimumab therapy (anamnesis, chest Rx, PPD skin test, IGRA test). Patients treated with anti-TNF- $\alpha$ have also an increased risk of viral infection, in particular of reactivation of chronic or latent infection. The hepatitis $\mathrm{B}$ virus and herpes family virus (HSV, EBV, CMV, VZS) are the most frequently involved, so it is very important to screen patients before starting therapy and to recognize any clinical manifestation. For patients with latent HBV infection, prophylaxis with lamivudine is recommended during anti-TNF- $\alpha$ therapy. ${ }^{38}$

Data on safety of adalimumab in UC patients are limited. No deaths or malignancies have been reported. In the induction $\mathrm{RCT},{ }^{33}$ serious adverse events occurred in 7.6\%, 3.8\%, and $4.0 \%$ of patients in the placebo, adalimumab $80 / 40 \mathrm{mg}$, and adalimumab 160/80 mg groups, respectively. In the study by Afif et al, ${ }^{28}$ serious adverse events were reported in six out of 20 patients, none of which were judged as probably related to the administration of adalimumab. Therefore the safety profile seems to be similar to the experience with this drug in CD.

As reported in a multicenter Italian cohort of IBD patients treated with biological drugs, patients older than 65 years have a higher rate of severe infections and mortality than younger patients or patients of the same age who did not receive these therapeutics. ${ }^{39}$

Finally, an emerging and increasing paradoxical collateral effect of anti-TNF- $\alpha$ drugs is psoriasis. Many cases have been reported of new-onset of palmo-plantar psoriasis occurring in the course of treatment from days to years after drug initiation, most of which resolve after cessation of the drugs or in some cases after switching to other anti-TNF- $\alpha$. The treatment (topical or systemic therapy) is based on tolerance of patients and extension of lesions. ${ }^{40}$

\section{Immunogenicity}

The fully human "designed" features of adalimumab do not eliminate the risk of antidrug antibodies. In fact, differences in amino acid sequences or glycoprotein content could induce an activation of adaptive immunity with production of human-antihuman antibodies (HAHA). In the CLASSIC 2 study $^{8}$ the percentage of patients developing antibodies to the human antibody adalimumab was low $(7 / 269,2.6 \%)$, all in the 185 patients without concomitant immunosuppression (3.8\%). Data on correlation between the presence of anti-adalimumab antibodies and adverse events are lacking. However, the long-term outcome of adalimumab therapy seems to be influenced by the presence of HAHA and the trough serum levels. Data from the cohort of Leuven University Hospital (168 CD patients, who previously failed infliximab) showed that low trough serum concentrations of adalimumab were associated with increased early and late discontinuation rates. HAHA were detected in $9.2 \%$ of the $92 \%$ of patients with trough serum concentration below the threshold for detection at least once during the follow-up, which may be responsible for rapid elimination of adalimumab and loss of response. However, there was no direct relationship between trough serum concentration and short-term efficacy of treatment. Concomitant immunomodulator therapy did not influence treatment outcome, adalimumab trough serum concentration, rate of adverse events, or development of HAHA. ${ }^{41}$ On the other hand, the chimeric features of infliximab (75\% human and $25 \%$ murine sequence) are correlated with development of antibodies to 
infliximab, which may also be a mechanism underlying the secondary loss of response to infliximab.

\section{Pregnancy and breast feeding}

Adalimumab is classified as pregnancy category B drug by the US Food and Drug Administration, which means that either animal studies have not demonstrated a fetal risk but there are no controlled studies in pregnant women, or animal studies have shown an adverse effect that was not confirmed in controlled studies in women in the first trimester. However, the recommendation to withdraw adalimumab before the third trimester, likewise infliximab, is arbitrary and based on the evidence that IgG1 passes the placental barrier during the second and the third trimester. Little data exist on the diffusion of adalimumab across the placenta. In a recent study, which includes five pregnant women with IBD receiving adalimumab, levels of the drug were measured in the mother, infant, and cord blood on the day of the birth. In all cases, the cord blood level of adalimumab was higher than the maternal level, supporting active transfer from the mother to the infant. Adalimumab also was detectable in the infant blood for at least 3 months from birth. ${ }^{42}$ Few data have been published on safety of adalimumab in pregnancy, especially in IBD patients. A report from the Organization for Teratology Information Specialists on 33 women enrolled in a prospective study on adalimumab use in pregnancy has shown a $12.1 \%$ rate of spontaneous abortion and stillbirth, not significantly different from the $5.6 \%$ of the diseased controls and $4 \%$ in the general population. Also congenital malformations and preterm deliveries were in the range of the nondisease controls. ${ }^{43}$ Similar results come from a recent systematic review of the literature on anti-TNF- $\alpha$ use and fetal risk, in which 38 studies were included, for a total number of 437 women (189 infliximab, 230 adalimumab, 18 certolizumab pegol). The rates of congenital abnormalities (3.4\%), fetal deaths (8.5\%), and preterm births $(2.7 \%)$ were similar to the rates in the general population. ${ }^{44}$ Safety during breastfeeding is unknown, but little evidence is available on excretion of adalimumab in breast milk. ${ }^{45}$

\section{Future perspectives}

Evidence from published research supports the fact that early treatment with anti-TNF- $\alpha$ seems to be more effective than traditional therapy for inducing clinical benefits and endoscopic remission, especially in selected patients with features at diagnosis that suggest poor outcome (top-down strategy). ${ }^{46} \mathrm{At}$ present, data on top-down strategies are not available for adalimumab. In addition, for infliximab, the combination therapy with azathioprine seems to be the most effective treatment for inducing steroid-free remission at 26 weeks. ${ }^{47}$ The first data on the comparison of the combination of adalimumab with azathioprine vs adalimumab as monotherapy are pending, ${ }^{48}$ but larger randomized controlled trials are necessary.

\section{Conclusion}

The advent of biological agents has modified the paradigm of treatment of IBD, since they are effective for induction and maintenance of sustained corticosteroid-free remission and mucosal healing. The current guidelines recommend that anti-TNF- $\alpha$ therapies should be reserved for IBD patients in whom conventional therapies have failed, as second-line therapy. In particular in CD, all the three anti-TNF- $\alpha$ available are recommended, in combination with surgical therapy, for patients with moderately-to-severely active disease, who have previously been steroid-refractory, -dependent, or -intolerant and with perianal disease. Adalimumab is an effective therapy for inducing and maintaining remission in patients with active moderate-to-severe luminal CD, naïve to anti-TNF or with intolerance or loss of response to infliximab, and in perianal fistulizing patients. It is well tolerated and not associated to adverse acute or delayed reactions. In the main clinical trials the rates of adverse events were comparable with other antiTNF- $\alpha$ and to other approved indications for adalimumab. The rates of induced antidrug antibodies were lower than for infliximab and this phenomenon was not associated with adverse events or loss of response. At present, because of the lack of comparative trials with different anti-TNF- $\alpha$ in CD, the decisions of clinicians are often determined case by case and based on personal experiences, not on objective criteria. Subcutaneous administration may be preferred by many patients because it allows home-based care.

In UC patients, there is an increasing evidence that adalimumab could be effective for inducing and maintaining remission, with variable action in different subsets of patients. Although the percentages of UC patients responding to adalimumab in the available studies are not outstanding, they are significantly higher than in the placebo groups. This is an important achievement, which will open the way for the use of a second anti-TNF- $\alpha$ agent in these patients, when for instance, the response to infliximab is lost or an infusion reaction develops. Furthermore, direct comparison of responder rate is not feasible among studies employing different drugs, with different designs and selection criteria.

Further efforts should be made to improve our knowledge so that in future every IBD patient could be treated with the right drug at the right time. 


\section{Disclosure}

The authors report no conflicts of interest in this work.

\section{References}

1. Baumgart DC, Carding SR. Inflammatory bowel disease: cause and immunobiology. Lancet. 2007;369:1627-1640.

2. Dignass A, Van Assche G, Lindsay JO, et al. The second European evidenced-based consensus on the diagnosis and management of Crohn's disease: current management. J Crohns Colitis. 2010;4:28-62.

3. Lichtenstein GR, Hanauer SB, Sandborn WJ. Practice Parameters Committee of the American College of Gastroenterology. Management of Crohn's disease in adults. Am J Gastroenterol. 2009;104:465-483.

4. Travis SP, Stange EF, Lemann L, et al. European evidenced-based consensus on the management of ulcerative colitis: current management. J Crohn's Colitis. 2008;2:24-62.

5. Kornbluth A, Sachar D, et al. Ulcerative Colitis Practice Guidelines in Adults: American College of Gastroenterology, Practice Parameters Committee. Am J Gastroenterol. 2010;105:501-523.

6. HUMIRA ${ }^{\circledR}$ Product monograph, Abbott Laboratories, Abbott Park, IL.

7. Hanauer SB, Sandborn WJ, Rutgeerts P, et al. Human anti-tumor necrosis factor monoclonal antibody (adalimumab) in Crohn's disease: the CLASSIC-I trial. Gastroenterology. 2006;130:323-333.

8. Sandborn WJ, Hanauer SB, Rutgeerts P, et al. Adalimumab for maintenance treatment of Crohn's disease: results of the CLASSIC II trial. Gut. 2007;56:1232-1239.

9. Colombel J-F, Sandborn WJ, Rutgeerts P, et al. Adalimumab for maintenance of clinical response and remission in patients with Crohn's disease: the CHARM trial. Gastroenterology. 2007;132:52-65.

10. Feagan BG, Panaccione R, Sandborn WJ, et al. Effects of adalimumab therapy on incidence of hospitalization and surgery in Crohn's disease: results from the CHARM study. Gastroenterology. 2008; 135:1493-1499.

11. Colombel JF, Sandborn WJ, Rutgeerts P, et al. Comparison of two adalimumab treatment schedule strategies for moderate-to-severe Crohn's disease: results from the CHARM trial. Am J Gastroenterol. 2009;104:1170-1179.

12. Sandborn WJ, Colombel JF, Schreiber S, et al. Dosage adjustment during long-term adalimumab treatment for Crohn's disease:clinical efficacy and pharmacoeconomics. Inflamm Bowel Dis. 2011;17:141-151.

13. Panaccione R, Colombel JF, Sandborn WJ, et al. Adalimumab sustains clinical remission and overall clinical benefit after 2 years of therapy for Crohn's disease. Aliment Pharmacol Ther. 2010;31:1296-1309.

14. Lofberg R, Louis E, Reinisch W, et al. Adalimumab produces clinical remission and reduces extraintestinal manifestations in Crohn's disease: results from CARE. Inflamm Bowel Dis. 2011. [Epub ahead of print].

15. Billioud V, Sandborn WJ, Peyrin-Biroulet L. Loss of response and need for Adalimumab dose intensification in Crohn's disease: a systematic review. Am J Gastroenterol. 2011;106:674-684.

16. Colombel JF, Schwarts DA, Sandborn WJ, et al. Adalimumab for the treatment of fistulas in patients with Crohn's disease. Gut. 2009; 58:940-948.

17. Lichtiger S, Binion DG, Wolf DC, et al. The CHOICE trial: adalimumab demonstrates safety, fistula healing, improved quality of life and increased work productivity in patients with Crohn's disease who failed prior infliximab therapy. Aliment Pharmacol Ther. 2010;32:1228-1239.

18. Sandborn WJ, Rutgeerts P, Enns R, et al. Adalimumab induction therapy for Crohn disease previously treated with infliximab: a randomized trial. Ann Intern Med. 2007;146:829-838.

19. Panaccione R, Sandborn WJ, D’Haens G, et al. Adalimumab maintains long-term remission in moderately to severely active Crohn's disease after infliximab failure: 1-year follow-up of GAIN trial. [abstract] Gastroenterology. 2008;134:A133.

20. Ma C, Panaccione R, Heitman SJ, et al. Systematic review: the shortterm and long-term efficacy of adalimumab following discontinuation of infliximab. Aliment Pharmacol Ther. 2009;15(30):977-986.
21. Colombel JF, Rutgeerts P, Sandborn WJ. Deep remission for adalimumab-treated patients with moderate to severe ileocolonic Crohn's disease: results from EXTEND [abstract]. J Crohns Colitis. 2010;4:S10.

22. Colombel JF, Rutgeerts P, Sandborn WJ. Deep remission predicts longterm outcomes for Adalimumab-treated patients with Crohn's disease: data from EXTEND [abstract]. Gut. 2010;59(Suppl 3):A80.

23. Rosh JR, Lerer T, Markowitz J, et al. Retrospective Evaluation of the Safety and Effect of Adalimumab Therapy (RESEAT) in pediatric crohn's disease. Am J Gastroenterol. 2009;104:3042-3049.

24. Russell RK, Wilson ML, Loganathan S, et al. A British Society of Paediatric Gastroenterology, Hepatology and Nutrition survey of the effectiveness and safety of adalimumab in children with inflammatory bowel disease. Aliment Pharmacol Ther. 2011;33:946-953.

25. Hyams JS, Griffiths AM, Markowitz J, et al. Induction and maintenance adalimumab therapy for the treatment of moderate to severe Crohn's disease in children [abstract]. Gastroenterology. 2011;140(Suppl 1): S90.

26. Peyrin-Biroulet L, Laclotte C, Roblin X, et al. Adalimumab induction therapy for ulcerative colitis with intolerance or lost response to infliximab: an open-label study. World J Gastroenterol. 2007; 13:2328-2332.

27. Oussalah A, Laclotte C, Chevaux JB, et al. Long-term outcome of adalimumab therapy for ulcerative colitis with intolerance or lost response to infliximab: a single-centre experience. Aliment Pharmacol Ther. 2008;28:966-972.

28. Afif W, Leighton JA, Hanauer SB, et al. Open-label study of adalimumab in patients with ulcerative colitis including those with prior loss of response or intolerance to infliximab. Inflamm Bowel Dis. 2009; 15:1302-1307.

29. Gies N, Kroeker KI, Wong K, et al. Treatment of ulcerative colitis with adalimumab or infliximab: long-term follow-up of a single-centre cohort. Aliment Pharmacol Ther. 2010;32:522-528.

30. Taxonera C, Estellés J, Blanco I, et al. Adalimumab for ulcerative colitis patients previously treated with infliximab: outcomes at short and long term and predictors of response. Aliment Pharmacol Ther. 2011;33:340-348.

31. Hudis N, Rajca B, Polyak S, et al. The outcome of active ulcerative colitis treated with adalimumab [abstract]. Gastroenterology. 2009;136:A661.

32. García-Bosch O, Aceituno M, Perez J, et al. Efficacy of adalimumab in the treatment of ulcerative colitis [abstract]. J Crohns Colitis. 2010;4:S55.

33. Reinisch W, Sandborn WJ, Hommes DW, et al. Adalimumab for induction of clinical remission in moderately to severely active ulcerative colitis: results of a randomised controlled trial. Gut. 2011;60:780-787.

34. Reinisch W, Sandborn WJ, Kumar A, et al. 52-week clinical efficacy with adalimumab in patients with moderately to severely active ulcerative colitis who failed corticosteroids and/or immunosoppresants [abstract]. J Crohns Colitis. 2011;5:S10.

35. Sandborn WJ, Van Assche G, Thakkar RB, et al. Adalimumab improves health-related quality of life for 52 weeks in patients with ulcerative colitis [abstract]. J Crohns Colitis. 2011;5:S87.

36. Sandborn WJ, Van Assche GA, Reinisch W, et al. Induction and maintenance of clinical remission by adalimumab in patients with moderate-to-severe ulcerative colitis [abstract]. Gastroenterology. 2011;140(5 Suppl 1):S123-S124.

37. Colombel JF, Sandborn WJ, Panaccione R, et al. Adalimumab safety in global clinical trials of patients with Crohn's disease. Inflamm Bowel Dis. 2009;15:1308-1319.

38. Shale MJ, Seow CH, Coffin S, et al. Review article: chronic viral infection in the anti-tumour necrosis factor therapy era in inflammatory bowel disease. Aliment Pharmacol Ther. 2010;31:20-34.

39. Cottone M, Kohn A, Daperno M, et al. Advanced age is an independent risk factor for severe infections and mortality in patients given antitumor necrosis factor therapy for inflammatory bowel disease. Clin Gastroenterol Hepatol. 2011;9:30-35.

40. Fiorino G, Allez M, Malesci A, et al. Review article: TNF $\alpha$ induced psoriasis in patients with inflammatory bowel disease. Aliment Pharmacol Ther. 2009;29:921-927. 
41. Karmiris K, Paintaud G, Noman M, et al. Influence of trough serum levels and immunogenicity on long-term outcome of adalimumab therapy in Crohn's disease. Gastroenterology. 2009;137:1628-1640.

42. Mahadevan U, Miller JK, Wolf DC. Adalimumab levels detected in cord blood and infants exposed in utero [abstract]. Gastroenterology. 2011;140(5 Suppl 1):S61-S62.

43. Johnson DL, Jones KL, Chambers CD, et al. Pregnancy outcome in women exposed to adalimumab: the OTIS autoimmune disease in pregnancy project [abstract]. Gastroenterology. 2009;136(5 Suppl 1):A27.

44. Marchioni RM, Kerner C, Lichtenstein GR. Anti-TNF therapy and fetal risk: a systematic review of the literature [abstract]. Gastroenterology. 2011;140(5 Suppl 1):S772.
45. Ben-Horin S, Yavzori M, Katz L, et al. Adalimumab level in breast milk of a nursing mother. Clin Gastroenterol Hepatol. 2010;8:475-476.

46. D'Haens G, Baert F, Van Assche G, et al. Belgian Inflammatory Bowel Disease Research Group; North-Holland Gut Club. Early combined immunosuppression or conventional management in patients with newly diagnosed Crohn's disease: an open randomised trial. Lancet. 2008;371:660-667.

47. Colombel JF, Sandborn WJ, Reinisch W, et al. Infliximab, azathioprine, or combination therapy for Crohn's disease. N Engl J Med. 2010; 362:1383-1395.

48. van der Valk ME, van Oijen MG, Siersema PD, et al. Crohn's disease patients treated with adalimumab benefit from co-treatment with immunomodulators: results from a nationwide study in the Netherlands [abstract]. Gastroenterology. 2011;140(5 Suppl 1):S264.

\section{Publish your work in this journal}

Clinical and Experimental Gastroenterology is an international, peerreviewed, open access journal, publishing all aspects of gastroenterology in the clinic and laboratory, including: Pathology, pathophysiology of gastrointestinal disease; Investigation and treatment of gastointestinal disease; Pharmacology of drugs used in the alimentary tract;
Immunology/genetics/genomics related to gastrointestinal disease. This journal is indexed on CAS. The manuscript management system is completely online and includes a very quick and fair peer-review system. Visit http://www.dovepress.com/testimonials.php to read real quotes from published authors.

Submit your manuscript here: http://www.dovepress.com/clinical-and-experimental-gastroenterology-journal 